\title{
Advances in Diagnosis and Management of Cutaneous Adverse Drug Reactions: Current and Future Trends
}

\author{
Neil H. Shear and Roni P. Dodiuk-Gad. ADIS 2019. Hardcover ISBN 978-981-13-1488-9, eBook \\ ISBN 978-981-13-1489-6
}

\section{Mark Lebwohl ${ }^{1}$}

Published online: 8 May 2019

C) Springer Nature Switzerland AG 2019

Advances in Diagnosis and Management of Cutaneous Adverse Drug Reactions is a superb, comprehensive, and clinically useful book on cutaneous drug reactions. The stated purpose of this book is to update clinicians who are faced with the patient with a suspected drug-related rash. The book accomplishes that purpose and much more.

The text engaged me, even with an early chapter that explains the mechanism of each kind of drug reaction. It makes clinically practical points explaining why certain treatments work and others do not, and explains why certain genetic associations and various drug reactions occur. The chapter on pharmacogenomics is excellent and, again, has great clinical utility; it is followed by very useful chapters on Stevens-Johnson syndrome/toxic epidermal necrolysis (SJS/ TEN), DReSS syndrome (drug reaction with eosinophilia and systemic symptoms), acute generalized exanthematous pustulosis (AGEP), and urticarial reactions to drugs. The chapter on SJS/TEN provides precise instructions on how to manage patients with this life-threatening condition and also has a useful table of both the top ten culprit drugs and common drugs not specifically associated with SJS/TEN.

Specific chapters are devoted to dermatologic reactions to cancer therapies, anti-HIV therapies, and anti-tuberculosis therapies. The chapter on dermatologic adverse reactions to cancer drugs has very useful tables on the types of reactions that occur and on the management of those reactions. There are also chapters on adverse reactions to drugs in pediatric populations and in the elderly.

Mark Lebwohl

lebwohl@aol.com

1 Kimberly and Eric J. Waldman Department of Dermatology, Icahn School of Medicine at Mount Sinai, New York, NY, USA
The closing chapters include in vitro and in vivo tests for adverse cutaneous reactions, histology of adverse cutaneous reactions, and, most importantly, a practical approach to the diagnosis and management of patients with cutaneous adverse drug reactions.

This is a clinically practical book I am sure I will turn to when faced with a possible drug reaction and especially severe cutaneous reactions. This is one of those books I'll have both in my office and at home because that's where it will be most useful.

Don't wait for a drug reaction to happen. Advances in Diagnosis and Management of Cutaneous Adverse Drug Reactions should be in every medical library and on every practicing dermatologist's shelf.

\section{Compliance with Ethical Standards}

Funding No sources of funding were used to assist in the preparation of this book review.

Conflict of interest Mark Lebwohl is an employee of Mount Sinai, which receives research funds from Abbvie, Amgen, AstraZeneca, Boehringer-Ingelheim, Celgene, Eli Lilly, Incyte, Janssen/Johnson \& Johnson, Kadmon, Leo Pharmaceuticals, Medimmune, Novartis, Pfizer, Sciderm, UCB, Ortho-dermatologics, and ViDac. Dr. Lebwohl is also a consultant for Allergan, Almirall, Arcutis, BoehringerIngelheim, Bristol-Myers Squibb, Castle Biosciences, LEO Pharma, Menlo, Mitsubishi Pharma, Neuroderm LTD, Pfizer, Promius/Dr. Reddy, Theravance Biopharma, and Verrica. 